\title{
PO3-2-19
}

Poster session

\section{Engagement of kappa opioid system in the right amygdala diminishes diffuse noxious inhibitory controls (DNIC)}

\author{
Megumi Ikegami, Kelsey Nation, Edita Navratilova, Daigo Ikegami, Frank Porreca \\ Department of Pharmacology, University of Arizona, College of Medicine, USA
}

Background: Diffuse noxious inhibitory control (DNIC) is known as pain modulatory system in which one painful stimulus inhibits another painful stimulus. We previously reported that the loss of DNIC observed in morphine-primed and stressed rats is ameliorated by kappa opioid receptor (KOR) antagonist injected into the central nucleus of amygdala $(\mathrm{CeN})$, suggesting that KOR-mediated descending facilitation from the $\mathrm{CeN}$ in morphine-primed rats masks the DNIC response. In this study, we invested if activation of kappa opioid system in the $\mathrm{CeN}$ in naive animals is sufficient to block the DNIC response, using designer receptor exclusively activated by designer drug (DREADDs) system.

Methods: Male and female KOR-Cre heterozygous mice and wild type littermates were used. DNIC response was measured by tail immersion as the test stimulus to elicit a tail flick response, and capsaicin was injected to the forepaw as the conditioning stimulus. To mimic KOR signaling selectively in the CeN, we injected Cre-dependent Gi-coupled designer receptor exclusively activated by designer drugs (Gi-DREADD) to the $\mathrm{CeN}$ of KOR-Cre heterozygous mice. On the test day, mice were systemically injected with clozapine- $\mathrm{N}$-oxide as a designer drug.

Results: DNIC response was observed in the tail flick test in control male and female mice, which had injected capsaicin into the forepaw. However, the DNIC response was not observed in both male and female KOR-Cre mice upon activation of Gi-coupled DREADD in the right CeN. In contrast, KOR-Cre mice expressing Gi-coupled DREADD in the left CeN still showed DNIC response in both of male and female. In addition, Gi-coupled DREADD injection in the right CeN caused hyperalgesia in female, but not male, KOR-Cre mice, in the tail-flick test without injection of capsaicin into the forepaw.

Conclusions: Our data suggest that activation of the same intracellular signaling cascades that are activated by KOR agonists in the right CeN is sufficient to cause a loss of DNIC likely through engagement of descending facilitation. There was little difference between male and female mice. Since it has previously been reported that the right amygdala, but not the left has a role in pain, our data supports this lateralization of amygdala function. 